\title{
Winter wheat in England and Wales, 1923-1995: what do indices of genetic diversity reveal?
}

\author{
C. S. Srinivasan ${ }^{1 *}$, Colin Thirtle ${ }^{2}$ and Paolo Palladino ${ }^{3}$ \\ ${ }^{1}$ Department of Agricultural and Food Economics, University of Reading, ${ }^{2}$ Department of \\ Environmental Science and Technology, Imperial College of Science, Technology and \\ Medicine, University of London and ${ }^{3}$ Department of History, Lancaster University, UK
}

Received 4 June 2002; Accepted 4 December 2003

\begin{abstract}
Genealogical data have been used very widely to construct indices with which to examine the contribution of plant breeding programmes to the maintenance and enhancement of genetic resources. In this paper we use such indices to examine changes in the genetic diversity of the winter wheat crop in England and Wales between 1923 and 1995. We find that, except for one period characterized by the dominance of imported varieties, the genetic diversity of the winter wheat crop has been remarkably stable. This agrees with many studies of plant breeding programmes elsewhere. However, underlying the stability of the winter wheat crop is accelerating varietal turnover without any significant diversification of the genetic resources used. Moreover, the changes we observe are more directly attributable to changes in the varietal shares of the area under winter wheat than to the genealogical relationship between the varieties sown. We argue, therefore, that while genealogical indices reflect how well plant breeders have retained and exploited the resources with which they started, these indices suffer from a critical limitation. They do not reflect the proportion of the available range of genetic resources which has been effectively utilized in the breeding programme: complex crosses of a given set of varieties can yield high indices, and yet disguise the loss (or non-utilization) of a large proportion of the available genetic diversity.
\end{abstract}

Keywords: coefficient of parentage; genetic diversity; plant breeding

\section{Introduction}

The transformation of plant breeding into an organized scientific activity over the last 100 years, involving the purposive incorporation of desirable traits into new varieties, has produced spectacular successes in terms of yield gain for a range of crops (Fuglie et al., 1996;

* Corresponding author: Department of Agricultural and Food Economics, School of Agriculture, Policy and Development, The University of Reading, Reading RG6 6AR, UK.

E-mail: aes02css@reading.ac.uk
Thirtle et al., 1998). But the very success of modern plant breeding has led to apprehensions about its potential adverse impact on the genetic diversity within farmers' fields. It could be argued that as farmers have adopted modern varieties over large areas for economic reasons, the genetic uniformity of agricultural crops may have increased considerably and some genetic resources may have been irretrievably lost. Erosion of genetic diversity can not only increase the vulnerability of agricultural crops to diseases and pests, but also affect the possibilities of developing new resistant varieties in the future. 
Modern plant breeding continues to be critically dependent on the diversity, continued availability and exchange of plant genetic resources (PGR). The key concern has been that the current thrust toward uniformity could undermine these critical resources (FAO, 1996; Swanson, 1997). One important reason for this concern is that the purposive incorporation of genetic diversity in new varieties requires the evaluation and screening of a large range of exotic germplasm. The development of genebanks with large collections of germplasm may improve the availability of material for plant breeders. At the same time, however, the time and costs associated with evaluation and the pressures for quicker development of new varieties may lead breeders to choose from a narrower range of tried and tested, 'elite' germplasm and consequently to the loss of unused genetic resources. Such loss could also be accentuated by the introduction of intellectual property rights (IPRs) for plant varieties in the following ways:

(i) Plant variety protection (PVP) systems stipulate that a new variety must be phenotypically 'distinct' from all other varieties in respect of one or more 'important' characteristics. Since the kind of differentiation that is required for protection is not precisely specified, it may lead breeders to resort to minimal differentiation, i.e. to 'cosmetic' breeding of elite germplasm. ${ }^{1}$ Cosmetic breeding could be used to undermine the protection afforded by PVP to a leading variety. Berlan and Lewontin (1986) argue that product differentiation through creation and sale of proprietary varieties is essential if seed companies are to remain competitive. In such a situation, the increase in the number of varieties offered for sale after PVP may not be an adequate measure of the increasing agronomic value of varieties, because, meanwhile, the objective of breeders has shifted from increasing farm productivity to that of 'giving sales arguments to the marketing departments of seed companies' (1986, p. 787). Cosmetic breeding could lead to a portfolio of varieties not significantly distinct from one another in terms of their genetic composition.

\footnotetext{
${ }^{1}$ The criterion of distinctness under PVP should normally promote greater differentiation between varieties. However, the incentives for cosmetic breeding arise from the fact that under PVP laws a variety, which is only marginally distinct from another variety, can qualify as a new variety in its own right and be protected. This allows the breeder of the 'new' variety to appropriate returns from the original innovation without much effort. The provisions relating to essentially derived varieties in the UPOV (1991) Convention (UPOV, 1994) are designed to discourage cosmetic breeding. But a clear technical definition of what constitutes an essentially derived variety is yet to emerge and these provisions are yet to be applied in practice.
}

(ii) PVP systems specify that a new variety must be "uniform' and 'stable' in order to qualify for protection. A plant variety innovation need not be necessarily uniform or stable in order to be economically useful. However, if IPRs are to be applied to plant varieties, they must be 'identifiable', i.e. distinguishable from other varieties. The uniformity and stability criteria are intended to facilitate identifiability. Therefore, the administrative requirement of identifiability in IPR systems creates an incentive for breeding uniform varieties that do not exhibit significant variations over repeated cycles of propagation.

(iii) Institutional changes in plant breeding have tended to favour 'centralized crop breeding' (Reid, 1992). IPRs enhance incentives to develop varieties that will have a large potential demand. To ensure maximum demand for their varieties, seed companies will tend to focus their research on commonly utilized high-value crops and develop varieties that can be cultivated as widely as possible. To do so means breeding through selection of genes for maximum adaptability while introducing new varieties. This IPR-supported bias toward centralized crop breeding could lead to biodiversity erosion through (i) decreased crop diversity and (ii) decreased spatial diversity. Temporal diversity could, however, increase if varieties are replaced every few years.

The argument that modern plant breeding reduces genetic diversity has been challenged. Smale (1997) has questioned the assertion that modern wheat breeding causes genetic narrowing, arguing that no causal relationship between the green revolution and genetic erosion can be established for bread wheat given the difficulties of measuring genetic erosion and demonstrating causality. The pattern of genetic variation in farmers' wheat fields has undoubtedly changed over the past 100-120 years with increasing cultivation of varieties released by plant breeding programmes, but the implications of these changes for the scarcity of useful genetic resources are unclear. Modern varieties have very complex pedigrees and incorporate germplasm from a very diverse range of sources often spread across several continents. Smale presents some evidence, using different indicators of genetic diversity that the genetic diversity of CIMMYTrelated (International Maize and Wheat Improvement Center) wheats has increased since the early years of the green revolution.

In this paper, we examine the diversity of winter wheat varieties in England and Wales between 1923 and 1995 using the same kind of indices. The beginning of this period corresponds with the establishment of the 
National Institute of Agricultural Botany (NIAB; 1919) and the collection of data on variety performance and national distribution. It also corresponds with the establishment of a more coherent national policy for a number of important plant breeding institutions, the John Innes Horticultural Institute (1910), the Plant Breeding Institute (1912) and the Welsh Plant Breeding Station (1919) (Thirtle et al., 1998). A major landmark came in 1964 when the UK enacted Plant Variety Protection legislation following its membership of the UPOV Convention. The scope for protection of intellectual property in plant varieties altered the incentives under which public plant breeding institutions worked. These changes may have been accentuated in the 1980s by the declining budgetary support for plant breeding institutions, forcing them to look upon IPRs as sources of revenue. At the same time, the UK's membership of the European Union and adherence to European seed marketing regulations (national listing, EU-wide listing, etc.) provided opportunities for the better exploitation and enforcement of IPRs. ${ }^{2}$ Therefore, the trends examined in this paper will also indicate the impact of these institutional changes on genetic diversity.

\section{Methods of measuring diversity}

One of the simplest measures of genetic diversity is the number of varieties in existence in a given geographical area at a given point in time. Such a measure does not take into account the relative abundance of individual varieties. If one variety is grown over a relatively large area, while several others are confined to small plots, then the count of varieties does not really capture diversity. It may be more useful to count the number of varieties that account for a given proportion of the total area under a crop. But even such a measure does not really capture diversity because the genetic relationship between varieties may be very close. It may be still more useful to include genealogical information

A coefficient of parentage (COP) is an indicator that uses pedigrees to estimate the extent of genetic similarity between two varieties. The COP between two varieties is defined as the probability that a random allele at a random locus in one individual is identical by descent to a random allele at the same locus in the other individual (Cox et al., 1985). It is a pair-wise comparison and its computation requires detailed pedigrees of all varieties.

\footnotetext{
2 Berlan and Lewontin (1986) argue that the 'catalogue' and 'seed certification', the two pillars of the European seed regulatory system, provide de facto appropriability for breeders even in the absence of formal PVP systems.
}

In the common algorithms used to compute COP values ( $r$ ), the following assumptions are generally made: (i) the original ancestors of the cultivar variety are unrelated $(r=0)$; (ii) a cultivar obtains equal genetic contributions from its parents; (iii) all ancestors and parental lines are homozygous and homogeneous; (iv) the COP between a selection from a cultivar and the cultivar is 0.75 ; (v) the COP between two selections from the same cultivar is $(0.75)^{2}=0.56$; $(\mathrm{vi})$ the COP of a cultivar with itself is 1 .

The above assumptions are restrictive and arbitrary. The consequent limitations of the COP measure are well understood. For instance, the assumption that the original ancestors of the cultivars are unrelated may not be correct from a biological point of view and may tend to bias the COP downward. The assumption that each parent contributes equally to the genetic make-up of the offspring may distort the genetic relationship between varieties. The impact of recurrent selection on allele frequencies in genotypes may not be adequately captured by the rule-of-thumb adopted. Besides, the genetic composition of a variety itself may not be stable over time owing to random genetic $\mathrm{drift}^{3}$ (Meng et al., 1998). Despite these limitations COPs have been used extensively in the economic analysis of diversity. With the advent of molecular methods, it is possible to estimate more precisely the degree of relatedness of ancestors and the contribution of parents to the offspring. This holds out the promise that, eventually, measures of diversity based on COPs will overcome many of these difficulties.

In an economic analysis of diversity, the coefficient of diversity is calculated as $1-$ COP. However, the COP is a pair-wise measure and analysis of diversity at the crop level requires the aggregation of pair-wise COPs into an average COP measure for all varieties. The method used to arrive at an average COP from the pair-wise values depends on the purpose for which it is to be used. To assess the progress of breeding programmes in incorporating genetic diversity, a simple average of the elements of the COP matrix may be sufficient. Such an average will indicate how closely related the varieties are and 1 - average COP ( 1 - ACOP) will be a corresponding indicator of diversity. It must be noted that such a measure will yield a positive value $(1 / n$ where $n$ is the number of varieties) even when all the varieties are completely unrelated. ${ }^{4}$ According to Solow and Polasky (1994), any measure of diversity should possess

\footnotetext{
${ }^{3}$ Changes in the genetic composition of a variety over time would, however, not affect the calculation of COPs.

${ }^{4}$ To overcome the problem of a positive value of average COP even when all the varieties are unrelated, the average of the off-diagonal elements of the COP matrix could be taken.
} 
the following fundamental properties: (i) diversity should not be decreased by the addition of a variety; (ii) diversity should not increase when we add a variety identical to one already included in the set; and (iii) diversity should not be decreased by increasing the genetic distance between varieties.

Solow and Polasky (1994) have proposed an index of species diversity based on the notion of genetic distance that fulfils these three criteria. Their measure can be calculated as:

$$
V(S)=e^{\prime} F^{-1} e
$$

where $V(S)$ is a measure of diversity of a set of varieties $S$; $V(S)$ is a function of $F$, a matrix of pair-wise distances and $e$ is a vector of $1 s$. Larger values of $F$ imply greater diversity. The matrix is made up of pair-wise coefficients $f_{j k}$ contained in the interval $(0,1)$.

$$
f_{j k}=\operatorname{Corr}\left(I_{j}, I_{k}\right)=f\left(d_{j k}\right)
$$

where $j, k$ index the varieties in the pairing.

The elements of the correlation matrix can be interpreted as the probability that two varieties share the same characteristics. The pair-wise COP values have been used as the $f_{j k}$ to compute the Solow-Polasky index for the set of English and Welsh wheat varieties.

The above measures, however, will not reflect the diversity in farmers' fields as they do not take into account the differential adoption of varieties by farmers. Some varieties developed by breeding programmes may not be adopted at all by farmers, while some others may have a dominant share of the market in certain years. The diversity seen on farmers' fields may be very different from the diversity within the set of varieties developed by the breeding programmes. To reflect diversity on farmers' fields, it is necessary to use a weighting scheme that takes into account the adoption of varieties by farmers. Several alternative weighting schemes are possible. The one which is most commonly used is a scheme based on the acreage under each variety. The area-weighted average COP is computed as:

$$
\text { W.COP }=a^{\prime} X a
$$

where $a^{\prime}$ is an $n \times 1$ vector of area shares $\left(\Sigma a_{i}=1\right)$ and $X$ is a $n \times n$ matrix of pair-wise COPs for $n$ varieties under consideration. The expression for the average COP can be expanded as:

$$
\mathrm{W} . \mathrm{COP}=\sum_{1}^{n} a_{i}^{2} \operatorname{COP}(i, i)+\sum_{i=1}^{n} a_{i} a_{j} \operatorname{COP}(i, j)
$$

where $i, j$ index varieties and $i \neq j$

$$
=\sum_{1}^{n} a_{i}^{2}+\sum_{i=1}^{n} a_{i} a_{j} \operatorname{COP}(i, j)
$$

as $\operatorname{COP}(i, i)=1$.
The W.COP can, thus, be seen as the sum of two components-the first component $\left(\Sigma a_{i}^{2}\right)$ reflects the impact of the proportion of area sown to different varieties and the second component $\left(\Sigma a_{i} a_{j} \operatorname{COP}(i, j)\right)$ is nothing but the sum of the pair-wise COPs weighted by the product of the respective area shares. For $n$ varieties, the lowest theoretical value of the first component $(1 / n)$ is reached when all the varieties are equally distributed (i.e. all the $a_{i}$ values are equal). As we move away from a uniform distribution of varieties, the value of $\Sigma a_{i}^{2}$ increases (reaching a maximum value of 1 when just one variety covers the entire area) and correspondingly the measure of diversity decreases.

The W.COP takes into account the loss of diversity due to varieties not being evenly distributed and this in turn can be attributed to the various economic and social factors that influence the adoption of different varieties by farmers. It has, however, the important disadvantage that the 'area effect' due to the distribution of varieties can dominate the effect due to the genetic similarity or dissimilarity between varieties. When there are a limited number of varieties in cultivation and their distribution is uneven (e.g. one or two cultivars dominate the total area), then the value of the W.COP index is not very different from the value which would obtain if we were to simply assume that all the varieties were completely unrelated (all the pair-wise COPs are zero). In this situation, the W.COP index tells us very little about the genetic similarity or dissimilarities between varieties and needs to be interpreted carefully.

The genetic diversity of winter wheat varieties in England and Wales between 1923 and 1995 has been assessed in this paper using the following indices: (i) the simple average of all the pair-wise COPs (ACOP); (ii) the average of the pair-wise COP of the top five varieties in each year $(\operatorname{ACOP}(5))$; (iii) the Solow-Polasky index; and (iv) the area-weighted average COP (W.COP).

\section{Data}

Genealogical information on all varieties was collected from various NIAB publications (Classified List of Cereal Varieties; Varieties in Trials; Cereal Variety Handbookvarious years). The pair-wise COPs were computed using the International Crop Improvement System (ICIS) software program developed by CIMMYT (CIMMYT, 1998). The acreage under different varieties for each of the years (1923-1995) has been estimated from seed certification data, occasionally published information and data provided by NIAB and the Home Grown Cereals Authority. 


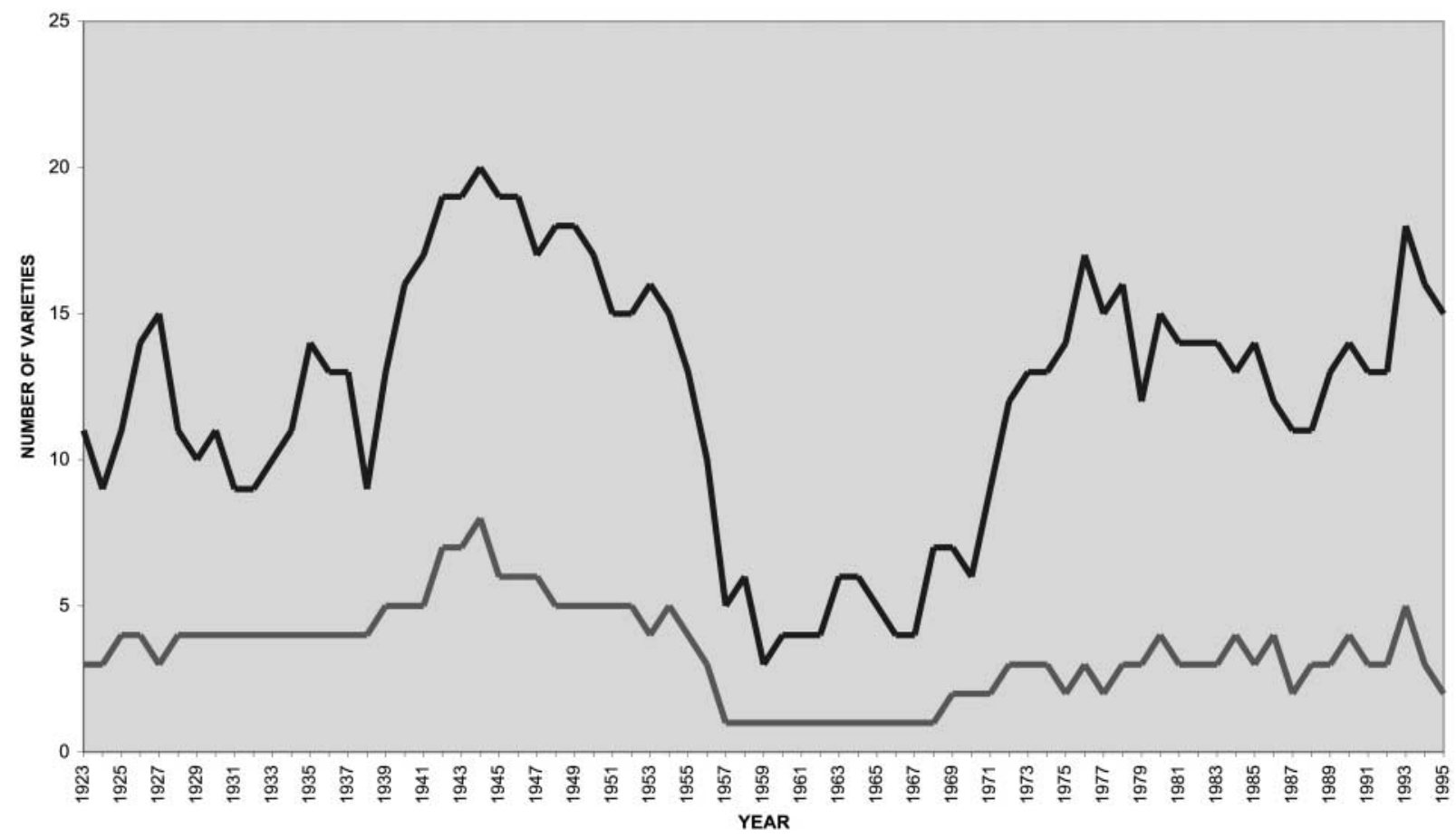

COUNT OF VARIETIES (AREA SHARE $>1 \%$ )

COUNT OF VARIETIES (Varieties accounting for $>50 \%$ area share)

Fig. 1. Count of UK wheat varieties.

\section{Results and discussion}

\section{Variety counts}

The number of varieties in each year since 1923 that accounted for $1 \%$ or more of the total area under wheat is shown in Fig. 1. The number of leading varieties, which together accounted for more than 50\% of the total area, is also shown alongside. For the 73-year period as a whole, farmers in England and Wales used an average of 12 varieties each year. The variety count ranged from a high of 20 in 1944 to a low of three in 1959. However, the high average of 12 varieties masks the fact that the number of leading varieties accounting for more than $50 \%$ of the area moved in a narrow range of one to four. For 10 years, from 1957 to 1967 , just one variety, 'Cappelle Desprez', accounted for more than $50 \%$ of the area. Starting with a portfolio of locally bred varieties in the 1920s, some of which survived for an extraordinary length of time, their numbers increased with the introduction of French varieties in the 1930s and the 1940s. As the French varieties became dominant, they almost completely displaced the locally bred ones and the number of varieties used by farmers declined sharply.

This unevenness in the distribution of varieties over the years has had a significant impact on the indices of genetic diversity. The decline was reversed only after the mid-1960s, almost coinciding with the introduction in 1964 of plant variety protection. From then on, the turnover of wheat varieties accelerated and varieties bred by the Plant Breeding Institute (e.g. 'Maris Huntsman') acquired significant market shares.

\section{Indices of genetic diversity}

The four COP measures are shown in Table 1 . The indices of diversity based on the simple average COP of all pairs of varieties, the average COP of five leading varieties and the Solow-Polasky index are plotted in Fig. 2, while the diversity measure based on area-weighted average COP is plotted in Fig. 3. For purposes of comparison with other indices whose values lie between 0 and 1 the values of the Solow-Polasky index have been divided by $10 .^{5}$

The most remarkable feature of all the indices of genetic diversity is the stability they exhibit over a period of 73 years. The indices that reflect breeding progress (ACOP and $\mathrm{ACOP}(5))$ move in a narrow range of $0.6-0.85$.

${ }^{5}$ Our main interest here is in the variability of the genetic diversity indices over time. Unlike the other indices whose values lie always between 0 and 1 , the values of the Solow-Polasky index can be greater than 1 (see Table 1). 
Table 1. COP measures and Solow-Polasky index

\begin{tabular}{|c|c|c|c|c|c|}
\hline Year & ACOP & $\mathrm{ACOP}(5)$ & $\begin{array}{c}\text { Solow-Polasky } \\
\text { index }\end{array}$ & W.COP & $\begin{array}{l}\text { Contribution of } \\
\text { variety distribution } \\
\text { to W.COP }(\%)\end{array}$ \\
\hline 1923 & 0.1560 & 0.2950 & 7.9328 & 0.2505 & 65.62 \\
\hline 1924 & 0.1590 & 0.2950 & 6.9135 & 0.2627 & 65.98 \\
\hline 1925 & 0.1270 & 0.2950 & 8.6892 & 0.2394 & 67.02 \\
\hline 1926 & 0.1141 & 0.2950 & 10.6904 & 0.2356 & 65.57 \\
\hline 1927 & 0.1361 & 0.2950 & 10.1657 & 0.2441 & 61.40 \\
\hline 1928 & 0.1477 & 0.2950 & 7.9574 & 0.2675 & 63.69 \\
\hline 1929 & 0.1687 & 0.2950 & 6.9574 & 0.2869 & 63.66 \\
\hline 1930 & 0.1704 & 0.2950 & 7.1578 & 0.2605 & 62.13 \\
\hline 1931 & 0.2298 & 0.2950 & 5.1578 & 0.2742 & 61.03 \\
\hline 1932 & 0.2022 & 0.2950 & 5.5575 & 0.2576 & 60.00 \\
\hline 1933 & 0.1737 & 0.2950 & 6.5574 & 0.2645 & 59.24 \\
\hline 1934 & 0.1818 & 0.3851 & 6.9574 & 0.2732 & 57.21 \\
\hline 1935 & 0.1886 & 0.3851 & 7.5574 & 0.2561 & 47.67 \\
\hline 1936 & 0.2128 & 0.3851 & 6.5574 & 0.2554 & 47.26 \\
\hline 1937 & 0.2128 & 0.3851 & 6.5574 & 0.2483 & 47.32 \\
\hline 1938 & 0.2747 & 0.3851 & 4.7128 & 0.2639 & 45.65 \\
\hline 1939 & 0.1918 & 0.3851 & 6.9791 & 0.2400 & 44.70 \\
\hline 1940 & 0.1610 & 0.3151 & 8.4296 & 0.2192 & 44.08 \\
\hline 1941 & 0.1396 & 0.3851 & 10.2450 & 0.2174 & 40.77 \\
\hline 1942 & 0.1242 & 0.3151 & 11.0312 & 0.1972 & 41.34 \\
\hline 1943 & 0.1236 & 0.3151 & 11.1213 & 0.1760 & 41.79 \\
\hline 1944 & 0.1183 & 0.3150 & 11.3055 & 0.1598 & 42.72 \\
\hline 1945 & 0.1224 & 0.3150 & 11.0846 & 0.1599 & 43.23 \\
\hline 1946 & 0.1445 & 0.2750 & 9.9617 & 0.1668 & 42.96 \\
\hline 1947 & 0.1377 & 0.1994 & 8.9255 & 0.1504 & 48.37 \\
\hline 1948 & 0.1651 & 0.3150 & 8.9255 & 0.1589 & 46.13 \\
\hline 1949 & 0.1373 & 0.2763 & 9.4790 & 0.1706 & 46.11 \\
\hline 1950 & 0.1788 & 0.2763 & 8.6995 & 0.1577 & 50.59 \\
\hline 1951 & 0.1646 & 0.2413 & 7.6170 & 0.1499 & 56.51 \\
\hline 1952 & 0.1795 & 0.3299 & 7.2770 & 0.1499 & 58.38 \\
\hline 1953 & 0.1680 & 0.2634 & 7.5418 & 0.1554 & 63.87 \\
\hline 1954 & 0.1728 & 0.2334 & 7.1620 & 0.1530 & 60.45 \\
\hline 1955 & 0.1720 & 0.3082 & 6.9454 & 0.1674 & 71.95 \\
\hline 1956 & 0.1544 & 0.3032 & 7.0816 & 0.2003 & 89.02 \\
\hline 1957 & 0.2224 & 0.2225 & 4.5118 & 0.3852 & 97.29 \\
\hline 1958 & 0.2286 & 0.2834 & 4.5891 & 0.6617 & 98.66 \\
\hline 1959 & 0.4680 & 0.4680 & 2.2397 & 0.7745 & 97.72 \\
\hline 1960 & 0.2696 & 0.2696 & 3.7180 & 0.6630 & 97.80 \\
\hline 1961 & 0.3021 & 0.3021 & 3.3412 & 0.5110 & 90.26 \\
\hline 1962 & 0.2798 & 0.2799 & 3.5855 & 0.6465 & 96.44 \\
\hline 1963 & 0.2001 & 0.2379 & 5.0724 & 0.6555 & 94.58 \\
\hline 1964 & 0.2440 & 0.2482 & 4.2949 & 0.6885 & 92.60 \\
\hline 1965 & 0.2992 & 0.2992 & 3.4934 & 0.7206 & 90.46 \\
\hline 1966 & 0.2768 & 0.2769 & 3.8046 & 0.5504 & 92.65 \\
\hline 1967 & 0.3701 & 0.3701 & 2.8046 & 0.6969 & 84.99 \\
\hline 1968 & 0.3286 & 0.4437 & 3.6552 & 0.6093 & 64.13 \\
\hline 1969 & 0.3901 & 0.4411 & 3.1305 & 0.5326 & 48.15 \\
\hline 1970 & 0.4126 & 0.4411 & 2.7970 & 0.5050 & 44.69 \\
\hline 1971 & 0.3135 & 0.4411 & 4.0572 & 0.4602 & 44.37 \\
\hline 1972 & 0.2405 & 0.3631 & 5.9967 & 0.3852 & 43.69 \\
\hline 1973 & 0.2364 & 0.4361 & 6.1916 & 0.3317 & 47.95 \\
\hline 1974 & 0.2268 & 0.3299 & 6.3038 & 0.2848 & 51.26 \\
\hline 1975 & 0.2301 & 0.2855 & 6.4904 & 0.3061 & 60.44 \\
\hline 1976 & 0.1786 & 0.3162 & 8.2846 & 0.2623 & 64.13 \\
\hline 1977 & 0.1547 & 0.2914 & 7.5765 & 0.2518 & 69.02 \\
\hline 1978 & 0.1416 & 0.2871 & 8.4341 & 0.2319 & 64.94 \\
\hline 1979 & 0.1658 & 0.2819 & 6.8127 & 0.2231 & 58.08 \\
\hline 1980 & 0.1526 & 0.2704 & 7.6553 & 0.1949 & 54.93 \\
\hline 1981 & 0.1384 & 0.2762 & 8.4265 & 0.2115 & 82.53 \\
\hline
\end{tabular}


Table 1. Continued.

\begin{tabular}{cccccc}
\hline Year & ACOP & ACOP(5) & $\begin{array}{c}\text { Solow-Polasky } \\
\text { index }\end{array}$ & W.COP & $\begin{array}{c}\text { Contribution of } \\
\text { variety distribution } \\
\text { to W.COP }(\%)\end{array}$ \\
\hline 1982 & 0.1580 & 0.2850 & 7.6519 & 0.1843 & 72.02 \\
1983 & 0.1472 & 0.3265 & 8.1130 & 0.1913 & 63.77 \\
1984 & 0.1303 & 0.2900 & 8.7222 & 0.1903 & 59.08 \\
1985 & 0.1333 & 0.2811 & 9.1461 & 0.1801 & 66.03 \\
1986 & 0.1682 & 0.2861 & 7.1395 & 0.1926 & 56.09 \\
1987 & 0.1856 & 0.2699 & 6.4807 & 0.2184 & 70.26 \\
1988 & 0.1468 & 0.2059 & 7.5510 & 0.1794 & 84.13 \\
1989 & 0.1353 & 0.2333 & 8.8873 & 0.1636 & 78.93 \\
1990 & 0.1710 & 0.2666 & 7.5462 & 0.1839 & 58.67 \\
1991 & 0.1691 & 0.2742 & 7.1215 & 0.2073 & 60.50 \\
1992 & 0.1678 & 0.3288 & 7.0760 & 0.2543 & 62.58 \\
1993 & 0.1430 & 0.2699 & 8.9783 & 0.1909 & 50.03 \\
1994 & 0.1515 & 0.3305 & 8.4256 & 0.2106 & 51.33 \\
1995 & 0.1767 & 0.3989 & 7.1833 & 0.2893 & 50.69 \\
\hline
\end{tabular}

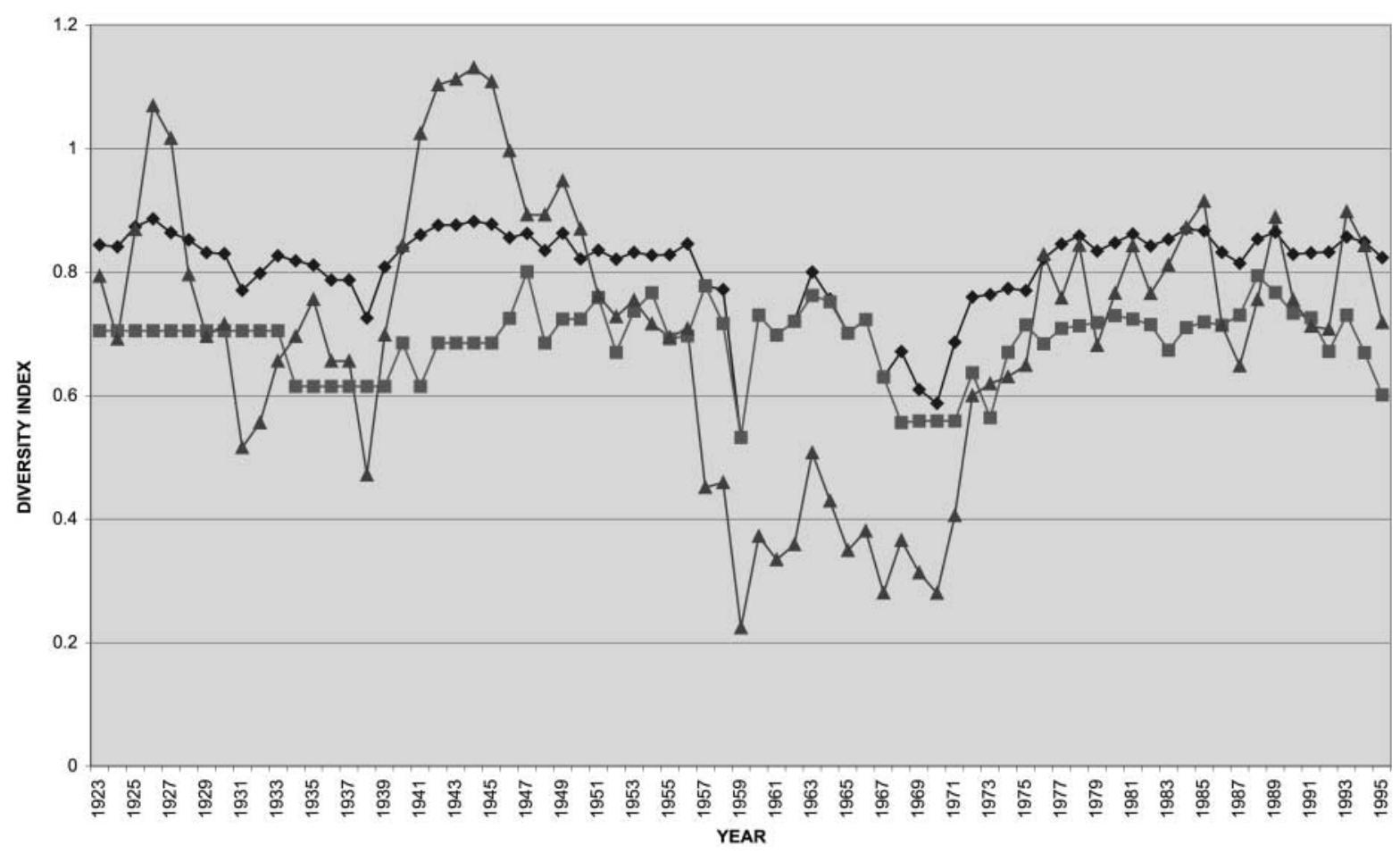

$\rightarrow-1-\mathrm{ACOP} \rightarrow-1-\mathrm{ACOP}(5) \multimap-$ Solow-Polasky Index

Fig. 2. Indices of genetic diversity breeding progress.

The simple average COP of the top five varieties shows even greater stability, reflecting the fact that for long stretches of time the portfolio of top varieties did not change at all. These trends provide no support for the view that modern plant breeding has led to a relative decline in genetic diversity. There is also no suggestion of a structural change following the introduction of plant variety rights. Thus, there is no reason to believe that varieties bred under the incentive of plant variety rights incorporate less genetic diversity than their predecessors. The recovery of genetic diversity indices after the mid-1960s (following their sharp fall in the 1950s) can be attributed to the large number of new varieties developed by public research institutions that acquired substantial market shares. It could, therefore, be argued that the institutional changes of the 1960s contributed to 


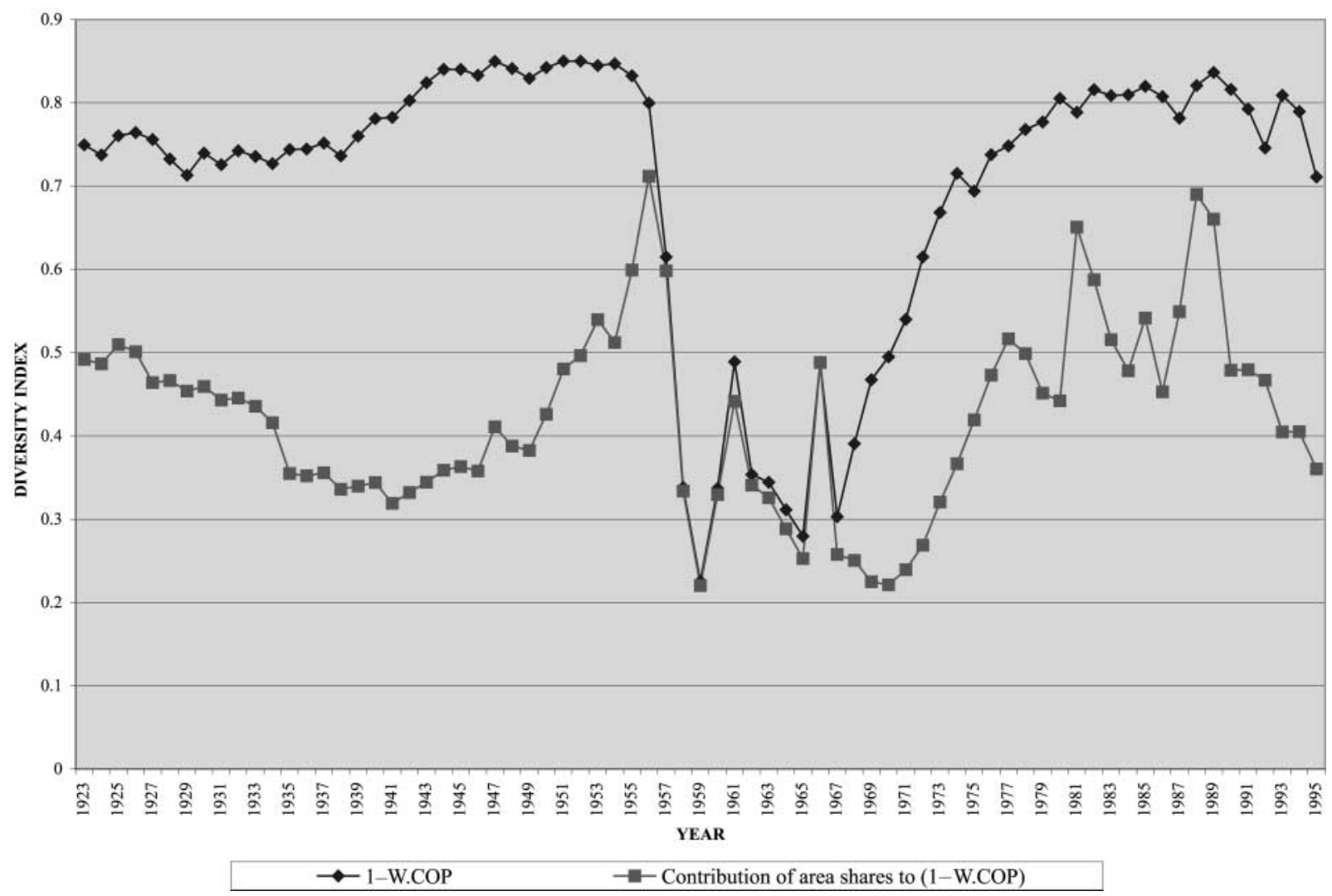

Fig. 3. Indices of genetic diversity on farmers' fields.

arresting the decline in genetic diversity witnessed previously.

However, when we look at the indices of diversity in farmers' fields, we find a dramatic decline in diversity indices from the late 1940 s to the early 1970s. This decline in diversity is not really due to the varieties in use being more closely related, but is instead due to the dominance of one or two varieties and the consequent decline in the number of varieties used by farmers. The diversity made available by the breeding programme was not reflected on farmers' fields as farmers chose to use only a limited number of varieties. The SolowPolasky index which is sensitive to the number of varieties in the set, and the area-weighted $1-$ W.COP index both registered a sharp decline during this period. The last column of Table 1 shows the contribution of the 'area effect' to the 1-W.COP index. This shows that when the number of varieties is small (e.g. less than five) the 'area effect' tends to dominate the index. That is, in this situation the value of the index is determined mainly by the evenness of distribution of varieties rather than by the degree of relatedness between varieties.

Importantly, the indices discussed above measure the diversity within the set of varieties for each year. What these indices track is the diversity retained in new varieties relative to the diversity available in the original set of ancestors. These indices give a fairly good idea of the extent to which breeders have retained and exploited the diversity in the original set of ancestors. They do not, however, indicate the extent to which the overall diversity available for a crop species has been exploited. Starting with a given set of varieties, it may be possible to maintain diversity by simply forming few complex combinations using a large number of those varieties but this may represent the exploitation of only a small proportion of the available diversity for the crop. Moreover, these indices provide no clue about the sources of germplasm (and its diversification). To assess the extent of diversification of genetic resources, we need to look at the relationship between varietal turnover and genetic diversity and analyse the pedigrees in terms of sources.

\section{Varietal turnover}

The rate of turnover of varieties can be an important determinant of genetic diversity over time. If new varieties replace older varieties more quickly, and the new varieties incorporate more diverse sources of germplasm, then the temporal diversity of the portfolio of 


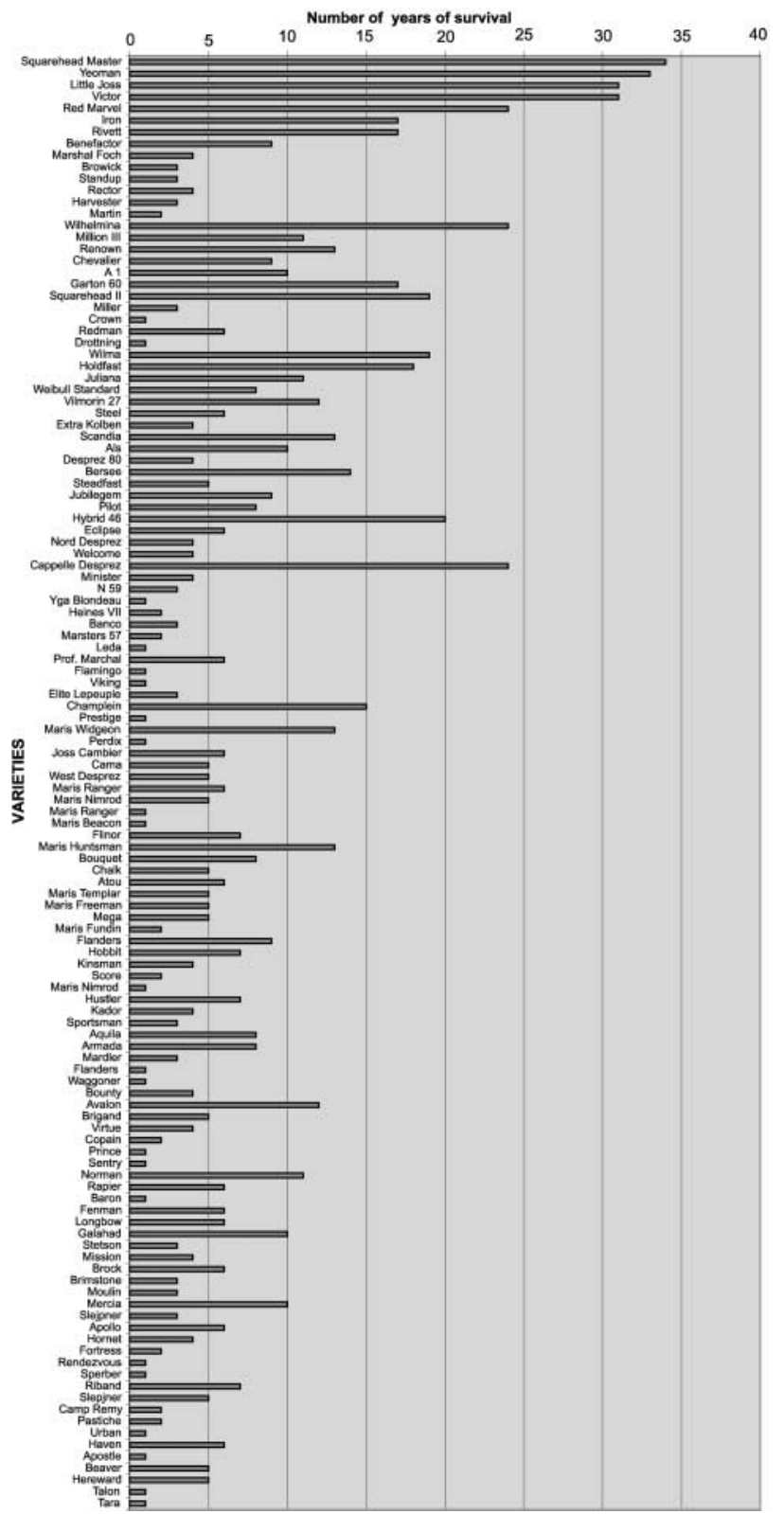

Fig. 4. Survival patterns of UK wheat varieties.

varieties can be expected to increase. The relationship between the turnover of varieties and genetic diversity can provide important pointers to the strategies followed by the breeding programme. The number of years for which different varieties survived is shown

\footnotetext{
${ }^{6}$ The data set on acreage shares spans the period 1923-1995. In Fig. 4, varieties which appeared in the National List up to 1991 are shown. In the case of recent varieties, which had not completed their commercial life by 1995, there is a downward bias in the number of years of survival. However, this affects only a limited number of varieties and does not alter the conclusion that recent varieties tend to survive for a much shorter time than the varieties of the 1940s, 1950s or 1960s.
}

in Fig. 4. In Fig. $4^{6}$ the varieties are arranged according to the year in which they first appeared in the portfolio of varieties.

Figure 4 reveals a dramatic decline in the survival of varieties. Starting in 1923 with varieties like 'Squarehead Master' and 'Yeoman' which survived for 35-40 years, the average number of years for which a variety survives has declined to less than 5 years in recent times. This trend is especially evident since the mid-1960s. This dramatic increase in the rate of turnover of varieties is reflected in the declining average age of the varietal portfolio (weighted average age of varieties in cultivation in any given year, with the share in acreage of each variety 


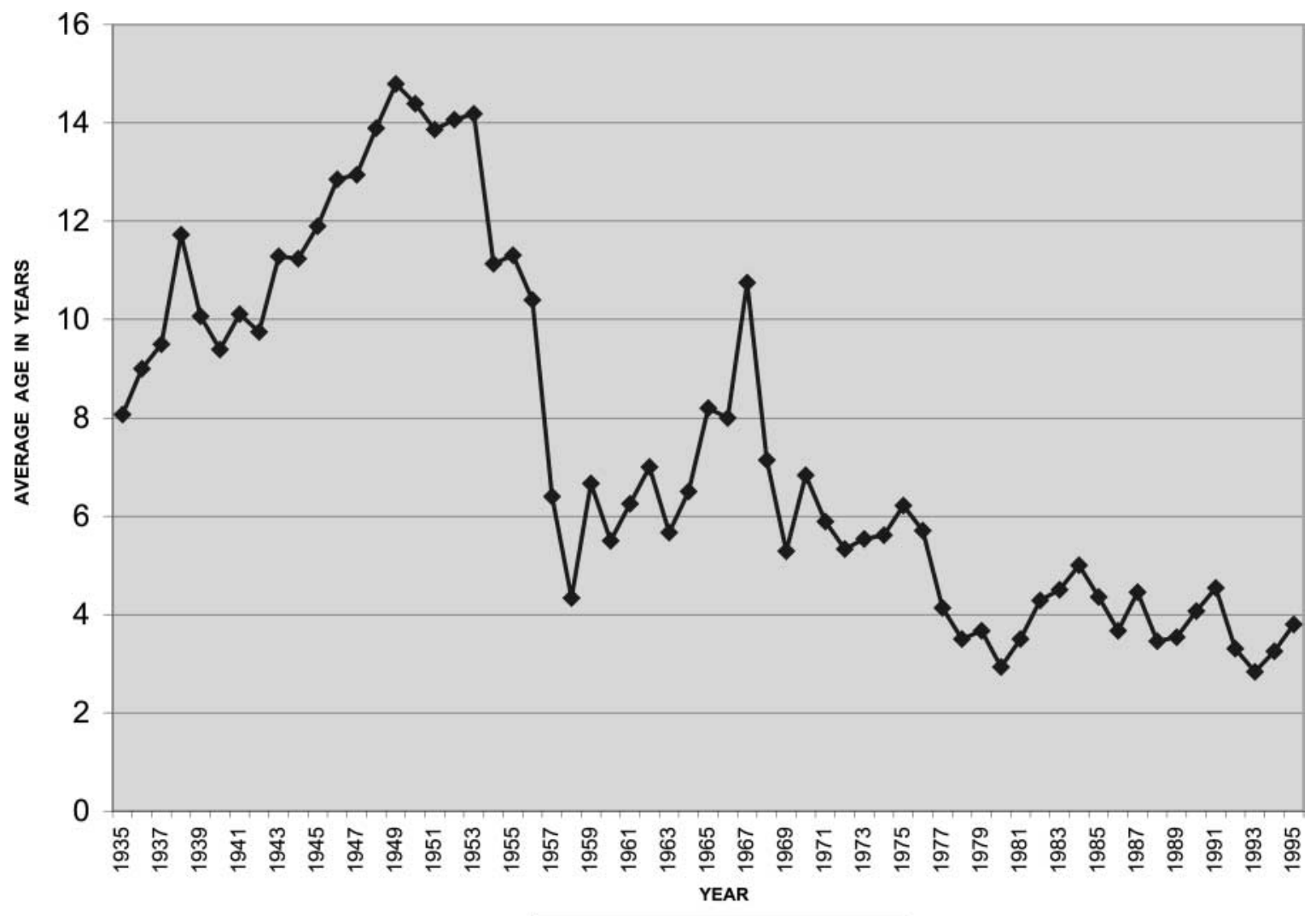

Fig. 5. Average age of UK wheat varieties.

being the weights), which has declined from about 14 years in the 1940s to 4 years in the 1990s (Fig. 5). Farmers have had access to a large number of quickly turningover new varieties since the mid-1960s.

It is noticeable that a more rapid turnover of varieties has not been associated with an increase in genetic diversity. This, however, provides no support to the argument that the large number of varieties developed in the post-PVP period mainly represents product differentiation by seed companies through 'cosmetic breeding'. If the varietal portfolio had a large number of varieties that were only marginally different from one another, then indices of genetic diversity based on genealogy would have certainly declined sharply. This has not been the case. It must also be noted that in the UK and other EU countries, new varieties can be marketed only if they are entered in the national catalogue after being tested for 'Value in Cultivation and Use' (VCU). Therefore, while a variety may become eligible for protection on the basis of (marginal) 'distinctness' it is unlikely to find a place in the national catalogue unless it also demonstrates some agronomic superiority or advantage. However, the stability of the indices in the context of a rapid turnover of varieties also indicates that the newer varieties developed since the 1960s have relied on a relatively unchanging parental pool. To put it differently, while newer varieties have been produced through novel combinations of a given set of parental varieties, there has been relatively limited introduction of new and hitherto unexploited material into the breeding programme. This suggests a lack of diversification in the sources of germplasm used.

Whether the lack of diversification of sources of germplasm was due to limited access to new sources of germplasm can be established by analysing the geographical provenance of parental varieties (and germplasm). It must be noted here that such an analysis, if based only on the immediate parents, may give a misleading picture. For instance, if a variety has two French parents it would be wrong to conclude that the variety is based on French material. The French parents may themselves incorporate material from very diverse sources. Therefore, it is necessary to go back several generations to assess the diversity of the sources of germplasm incorporated in a variety. ${ }^{7}$ Such an analysis has been attempted for the winter wheats in England and Wales in Table 2.

\footnotetext{
${ }^{7}$ On the other hand, it is not very useful to go back too many generations as the contribution of unknown varieties then becomes large and confounding.
} 


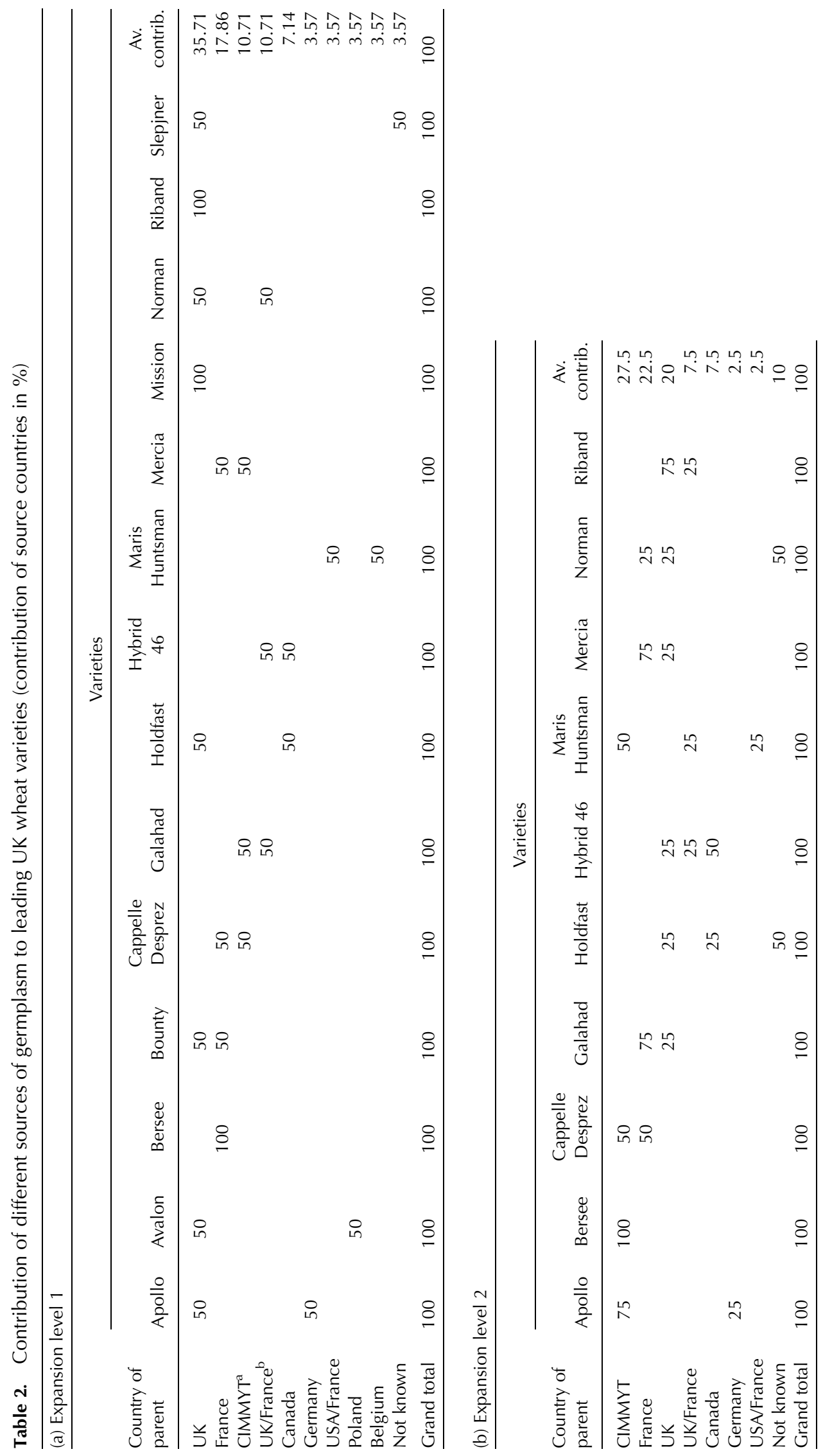




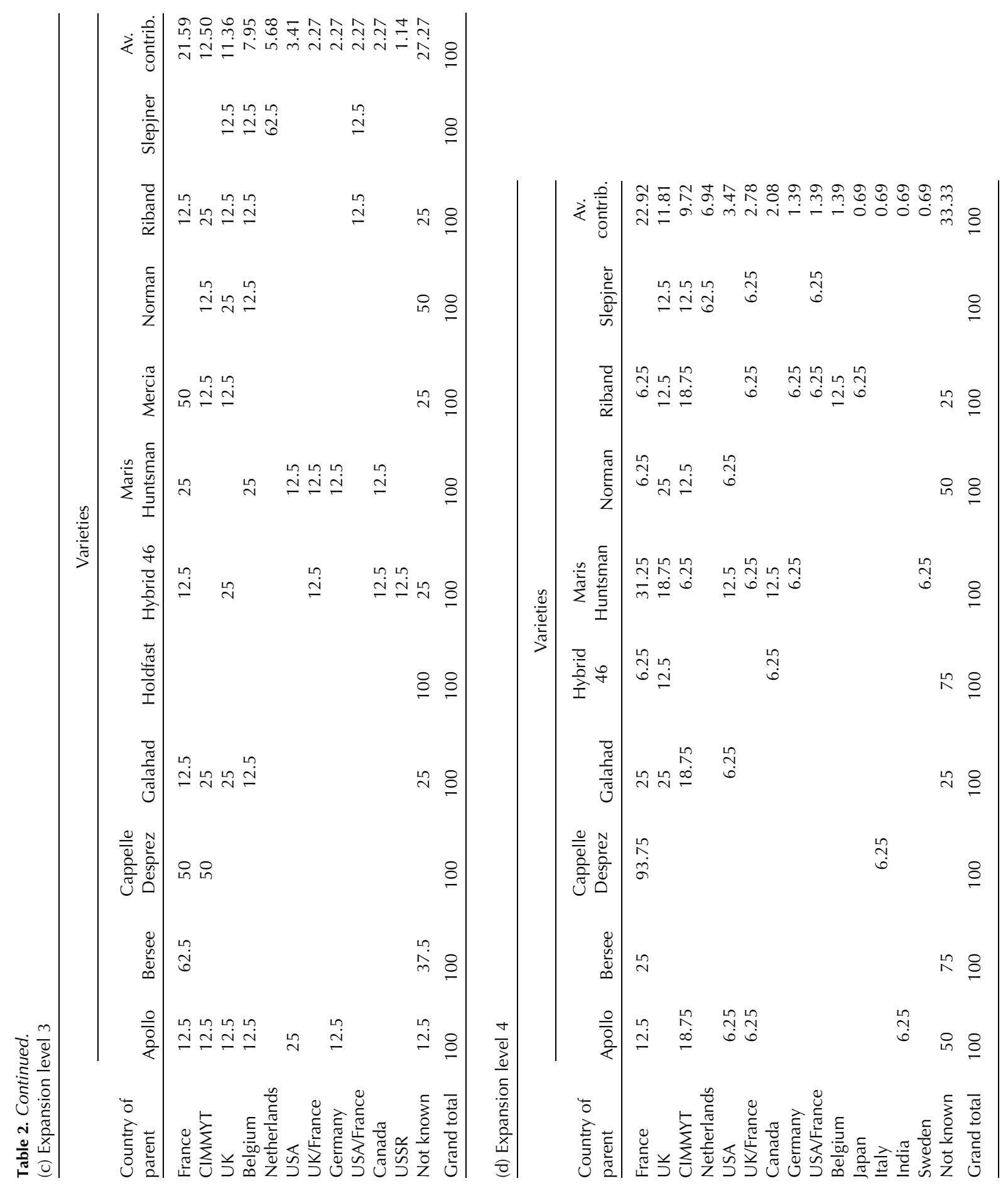




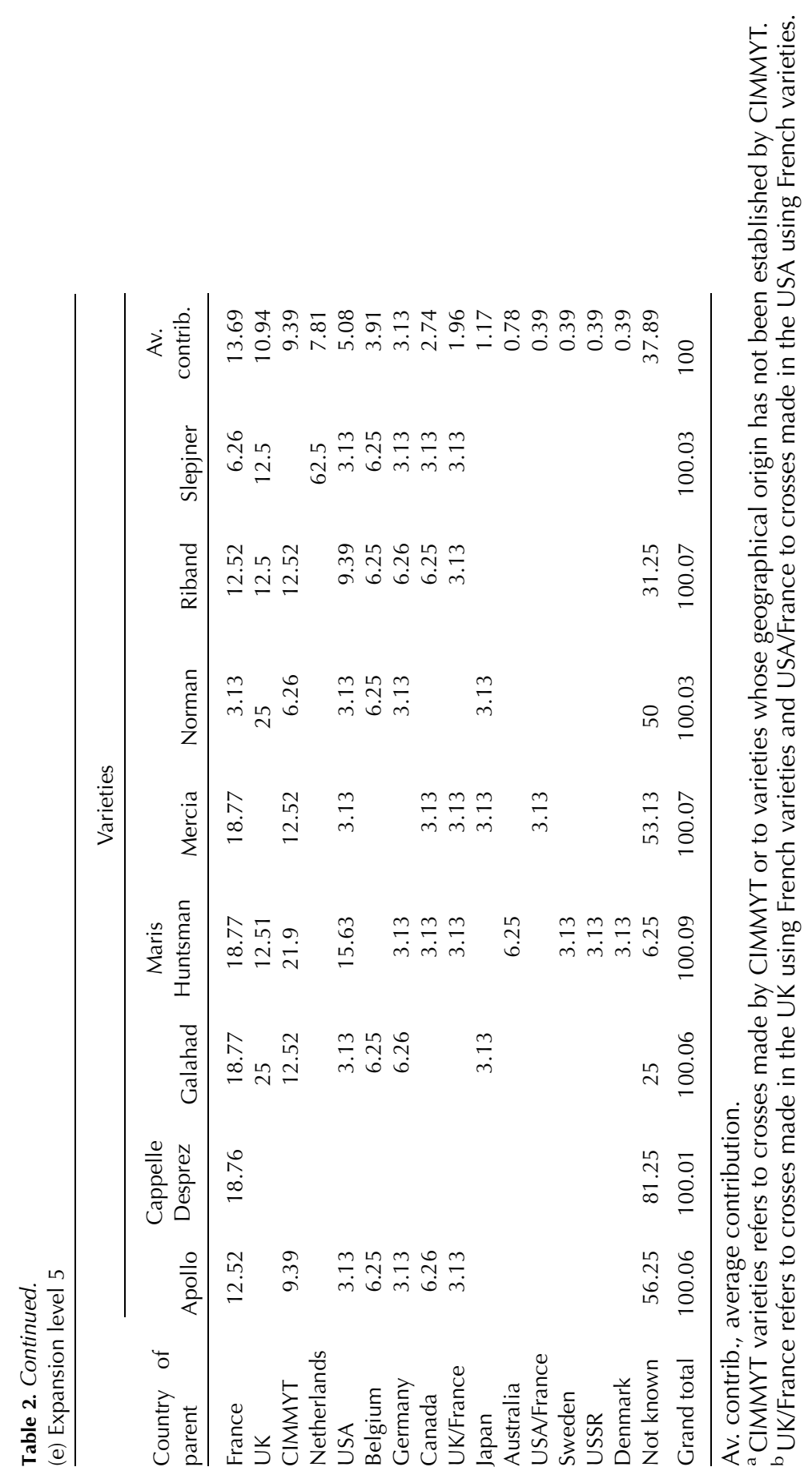


The geographical contribution has been assessed for different expansions of pedigrees, from one generation to five generations. An analysis of the immediate parentage reveals that almost all the leading UK varieties have been based on crosses of parental material from a fairly narrow range of geographical sources. Most are based on local, French (or local crosses made from French varieties), American/Canadian/Australian, and a sprinkling of other European varieties. The interesting feature that emerges from this analysis is that even as we expand the pedigrees to five generations, the composition of the sources of parental material does not change significantly. French varieties continue to remain fairly dominant and the contribution of varieties from the USA, Canada and Europe increases. This lends considerable support to the view that the wheat breeding programme in England and Wales has been based on material from a relatively narrow geographical range. A part of the explanation for this may be that only varieties from a similar agro-ecological area may prove useful in a breeding programme. Nevertheless, this is surprising considering CIMMYT appears to use a much more diversified range of germplasm.

The key result of this paper-that diversity of winter wheat varieties has been maintained relative to the original pool of ancestors, but there has been no substantial diversification of the sources of germplasm-is confirmed by a detailed analysis of biochemical and molecular markers (Donini et al., 2000). The study found that British varieties form a distinct cluster from the cluster of the 'rest-of-the-world' varieties. Moreover, varieties released in the later decades of the period studied in this paper include a significant amount of the germplasm, which had always been available. But there were large pools of diversity that had not been exploited in the UK wheat breeding programme. Therefore, studies based on molecular markers appear to confirm the finding that the development of new UK wheat varieties has relied on a relatively unchanging parental pool of varieties.

The limited diversification of winter wheat in England and Wales may have implications for its vulnerability to diseases like rust. CIMMYT seeks to address such problems by accumulating genes for resistance from diverse sources (Smale and McBride, 1996). Whether this is necessary remains to be demonstrated. It would, therefore, be useful to examine how the susceptibility of winter wheat in England and Wales has been affected by the geographical distribution of parental material. There may be pointers here for the future evolution of plant breeding strategies. It must be remembered, however, that the present analysis has demonstrated important limitations of genealogical indices for any discussion over the utilization of genetic resources.

\section{Conclusions}

Seventy-five years of organized winter wheat breeding in England and Wales have produced a steady stream of new varieties that have provided impressive yield gains. The institutional changes after the mid-1960s, especially the introduction of plant variety protection, have led to an accelerated turnover of varieties. Throughout this period, however, indices of genetic diversity based on genealogical information have shown remarkable stability, except during one short interlude characterized by the dominance of foreign varieties. These institutional changes, in fact, have had a generally positive effect on genetic diversity of wheat varieties in the UK. When we consider the entire period spanned by the data set, there is no evidence to suggest that the post-PVP period has been associated with lower levels of genetic diversity.

Empirical evidence for UK wheat does not support the view that the large number of varieties developed in the post-PVP period merely represent a proliferation of closely related varieties resulting from the product-differentiation efforts of seed companies in their quest for a larger market share. However, the stability of the indices in the context of an accelerated turnover of varieties does indicate a lack of diversification in the (geographical) sources of germplasm used in breeding programmes. Genetic diversity has been maintained mainly through more intensive use of a relatively unchanging germplasm pool. Plant breeders have been remarkably adept at exploiting the original range of diversity that was available to them. But the quicker replacement of varieties has not led, as might have been expected, to genetic diversification through incorporation of previously unexplored germplasm. This may have implications for the potential vulnerability of wheat varieties to disease and the future strategies of the breeding programme.

More importantly, the results for winter wheat in England and Wales show considerable caution must be exercised in using genealogical indices to assess the progress of conservation or utilization of genetic diversity. Firstly, complex crosses of a given set of varieties can yield high values, and yet disguise the loss (or nonutilization) of a large proportion of the available genetic diversity. Secondly, even when these indices are combined with data about varietal distribution to assess the impact of farmers' choices about which varieties to cultivate on genetic diversity, the combined values are more sensitive to changes in varietal shares than to the genealogical relationship between the varieties sown. In sum, high values of indices of genetic diversity, as they are currently articulated, should not be a cause for complacency regarding the maintenance and enhancement of plant genetic resources. 


\section{Acknowledgements}

Earlier versions of this paper were presented at the GCTE Conference, University of Reading, September 1999 and at the National Institute of Agricultural Botany in July 2000. We thank those who attended for their comments. We are also very grateful to the two anonymous referees for their comments and suggestions.

\section{References}

Berlan J-P and Lewontin R (1986) Breeders' rights and patenting life forms. Nature 322: 785-788.

CIMMYT (1998) International Crop Information System (ICIS) (computer program). Mexico: CIMMYT (International Maize and Wheat Improvement Center).

Cox TS, Lookhart GL, Walker DE, Harrell LG, Albers LD and Rodgers DM (1985) Genetic relationships among hard red winter wheat cultivars as evaluated by pedigree analysis and gliadin polyacrylamide gel electrophoretic patterns. Crop Science 25: 1058-1063.

Donini P, Law JR, Koebner RMD and Reeves JC (2000) Temporal trends in the diversity of UK wheat. Theoretical and Applied Genetics 100: 912-917.

FAO (Food and Agriculture Organization) (1996) Options for Access to Plant Genetic Resources and Equitable Sharing of their Use. Document prepared by the International Plant Genetic Resources Institute for the FAO's Commission on Genetic Resources for Food and Agriculture. CGRFAEX3/96/LIM/2. Rome.

Fuglie K, Ballenger N, Day K, Klotz C, Ollinger M, Reilly J, Vasavada U and Yee J (1996) Agricultural Research and Development: Public and Private Investments Under Alternative Markets and Institutions. Agricultural Economics
Report No. 735. Washington, DC: Economic Research Service, United States Department of Agriculture.

Meng ECH, Smale M, Bellon M, and Grimanelli D (1998) Definition and measurement of crop diversity for economic analysis. In: Smale M (editor) Farmers, Gene Banks and Crop Breeding: Economic Analysis of Diversity in Wheat, Rice and Maize. Dordrecht: Kluwer Academic Publishers, pp. 19-32.

Reid WV (1992) Genetic Resources and Sustainable Agriculture: Creating Incentives for Local Innovation and Adaptation. Biopiracy International No. 2. Nairobi and Maastricht: African Centre for Technology Studies.

Smale M (1997) The Green Revolution and wheat genetic diversity: some unfounded assumptions. World Development 25: $1257-1269$.

Smale M and McBride T (1996) Understanding global trends in the use of wheat diversity and international flows of wheat genetic resources. Part 1 of CIMMYT 1995/96 World Wheat Facts and Trends: Understanding Global Trends in the Use of Wheat Diversity and International Flows of Wheat Genetic Resources. Mexico: CIMMYT.

Solow A and Polasky S (1994) Measuring Biological Diversity. Woods Hole, MA: Woods Hole Oceanographic Institution.

Swanson T (1997) Global Action for Biodiversity: An International Framework for Implementing the Convention on Biodiversity. London: Earthscan Publications.

Thirtle C, Bottomley P, Palladino P and Schimmelpfennig DE (1998) The rise and fall of public sector plant breeding in the UK: a recursive model of basic and applied research, and diffusion. Agricultural Economics 19(1/2): $127-143$.

UPOV (International Union for the Protection of New Varieties of Plants; Union Internationale pour la Protection des Obtentions Végétales) (1994) International Convention for the Protection of New Varieties of Plants of December 2, 1961 as Revised at Geneva on November 10, 1972, and on October 23, 1978, and on March 19, 1991. Geneva: UPOV. 